\title{
Routine biochemistry in suspected vitamin D deficiency
}

\author{
Steven R Peacey MD FRCP
}

J R Soc Med 2004;97:322-325

SUMMARY

Vitamin D deficiency, which continues to be widespread amongst persons of Asian descent in the UK, is often detected from abnormal results on routine biochemistry. The aim of this study was to assess the frequency of abnormal results from routine baseline tests of serum calcium, phosphate, and alkaline phosphatase in patients who subsequently proved to have vitamin D deficiency and secondary hyperparathyroidism. A retrospective examination was undertaken to assess these baseline indices in a cohort of 84 such patients seen in Bradford-5 male; 80 of Asian descent; median age 46 years (range 16-82); serum 25-hydroxyvitamin $D<10 \mu g / L$; parathyroid hormone $>54 \mathrm{ng} / \mathrm{L}$.

Calcium was normal in 55 patients (66\%), phosphate in $68(81 \%)$ and alkaline phosphatase in 24 (29\%). In only 5 patients were all three indices outside the normal range. The median parathyroid hormone concentration was significantly greater in patients with abnormal routine biochemistry (145 [range 55-1662] ng/L) than in patients with normal routine biochemistry (88 [59-322] ng/L) but the median 25-hydroxyvitamin D levels did not differ (3.1 [1.3-9.9] and 3.0 [1.5-7.3] $\mu \mathrm{g} / \mathrm{L})$. Routine biochemistry was normal in $20 \%$ of cases.

If routine biochemistry is relied upon to detect vitamin D deficiency and osteomalacia, a substantial minority of cases will be missed.

\section{INTRODUCTION}

The high prevalence of vitamin D deficiency and osteomalacia within the Asian community in the UK has been amply documented since the 1960s. ${ }^{1-4}$ In 1976 Ford and co-workers, having studied the vitamin D status of Bradford's Asian children, wrote of the urgent need for prophylactic measures. ${ }^{3}$ Nevertheless, local awareness of this condition remains poor and sizeable numbers of patients with clinically severe vitamin D deficiency and osteomalacia continue to be seen in secondary care. To address this major public health issue the UK Department of Health opted for a strategy that relies on enhancement of professional awareness, health education, and vitamin D supplementation for particular groups rather than widespread fortification of foods. ${ }^{5,6}$ In practice, reliance is often on case finding, with a low threshold for considering vitamin $\mathrm{D}$ deficiency and osteomalacia in patients of Asian descent who present with musculoskeletal symptoms. In addition, the 'routine biochemistry' sought by primary care practitioners includes serum adjusted calcium $(\mathrm{Ca})$, phosphate (PO) and total alkaline phosphatase (ALP), often in conjunction with 25-hydroxyvitamin D (25-OHD). The last is particularly expensive.

Department of Diabetes and Endocrinology, Bradford Teaching Hospitals NHS Trust, Duckworth Lane, Bradford, West Yorkshire BD9 6RJ, UK
Whilst previous studies have examined biochemical indicators in patients with histological osteomalacia, the aim of the present investigation was to determine the frequency of normal and abnormal 'routine biochemistry' in a welldefined group of patients who were shown to have 25-OHD deficiency and secondary hyperparathyroidism.

\section{PATIENTS AND METHODS}

In 1999 a computerized database (Access, Microsoft Corp, USA) was established within the Department of Diabetes and Endocrinology to record data prospectively on all patients with a definite endocrine diagnosis. A retrospective search of this database was made to identify patients with a diagnosis of vitamin D deficiency or osteomalacia. Patients included in this cohort had been referred either because of a biochemical abnormality detected by their general practitioner or because in other secondary-care settings (endocrine, diabetes, general medicine) they had reported musculoskeletal symptoms. 116 patients were identified. 32 of these were excluded from further analysis because of inadequate biochemical data $(n=10)$, concomitant primary/ tertiary hyperparathyroidism (hypercalcaemia) $(n=7)$, diagnostic label of hypoparathyroidism incorrect $(n=4)$, renal impairment $(n=6)$, liver disease $(n=1)$, phenytoin therapy $(n=2)$ and pretreatment with vitamin D $(n=2)$. The remaining 84 patients all had their 25-OHD, parathyroid 
hormone $(\mathrm{PTH}), \mathrm{Ca}, \mathrm{PO}$ and ALP measured before treatment, and subsequent analysis is restricted to this group. All 84 had both hypovitaminosis $\mathrm{D}(<10 \mu \mathrm{g} / \mathrm{L})$ and a raised PTH $(>54 \mathrm{ng} / \mathrm{L})$. The timing of the samples proved to be evenly distributed throughout the year.

The Mann-Whitney U test was used for unpaired nonparametric data and Spearman's rank correlation test to look for associations. A $P$ value of $<0.05$ was taken as statistically significant. Local laboratory normal ranges were used as indicated. PTH was measured by whole-molecule double-antibody immunoassay (Nichols Advantage), 25 OHD by manual extraction single-antibody radioimmunoassay (DiaSorin, Stillwater, Minnesota), Ca by colorimetric-cresolphthalein complexone (Roche Hitachi 747), ALP by IFCC method, DEA buffer (Roche Hitachi 747), and PO by a colorimetric-phosphomolybdate method (Roche Hitachi 747). All assays were performed according to the manufacturers' instructions. Laboratory reference ranges for these indices were: $25-\mathrm{OHD} 10-45 \mu \mathrm{g} / \mathrm{L}, \mathrm{PTH}$ 10-54 ng/L, Ca $2.15-2.55 \mathrm{mmol} / \mathrm{L}, \mathrm{PO} 0.8-1.3 \mathrm{mmol} / \mathrm{L}$, ALP 50-240 IU/L.

\section{RESULTS}

79 of the 84 patients were female; 80 were of Asian descent and 4 European. The median age at diagnosis was 46 years (range 16-82). Median (range) serum 25-OHD was 3.1 (1.3-9.9) $\mu \mathrm{g} / \mathrm{L}, \quad$ PTH 117 (55-1662) ng/L, Ca 2.20 $(1.14-2.44) \mathrm{mmol} / \mathrm{L}, \quad$ PO $1.01 \quad(0.45-1.56) \mathrm{mmol} / \mathrm{L}$, ALP 286 (91-2863) IU/L.

Ca was normal in 55 (66\%), PO normal in 68 (81\%) and ALP normal in $24(29 \%)$ patients (Figure $1 a-c$ ). In 17 $(20 \%)$ patients, $\mathrm{Ca}, \mathrm{PO}$ and ALP were all within the normal range. Only $5(6 \%)$ patients had all three biochemical indices outside the normal range. The most frequent combined abnormality was low calcium and raised ALP (with or without abnormal PO), present in 24 (29\%) patients, and the most frequent isolated abnormality was a raised ALP, present in 27 (32\%) patients. Only 2\% of patients had a low $\mathrm{PO}$ in isolation.

Patients with normal biochemistry (Ca, PO and ALP), termed group $\mathrm{N}(n=17)$, were compared with patients in whom there was at least one abnormality (low $\mathrm{Ca}$, low PO or raised ALP), termed group ABN $(n=67)$. The PTH concentration was significantly greater in group ABN (145 [range 55-1662] ng/L) than in group N (88 [59-322] ng/L; $\mathrm{P}<0.002$ ) (Figure 2a). 25-OHD did not differ between group ABN (3.1 [1.3-9.9] $\mu \mathrm{g} / \mathrm{L})$ and group $\mathrm{N}(3.0[1.5-$ 7.3] $\mu \mathrm{g} / \mathrm{L}$ ); $P=0.96$ (Figure $2 b$ ). A negative correlation was found between PTH and calcium $(r=-0.57,-0.7$ to $-0.4 ; P<0.0001)$ and a positive correlation between PTH and $\operatorname{ALP}(r=0.6,0.45$ to $0.72 ; P<0.0001)$. Overall, a weak negative correlation was found between PTH and 25-
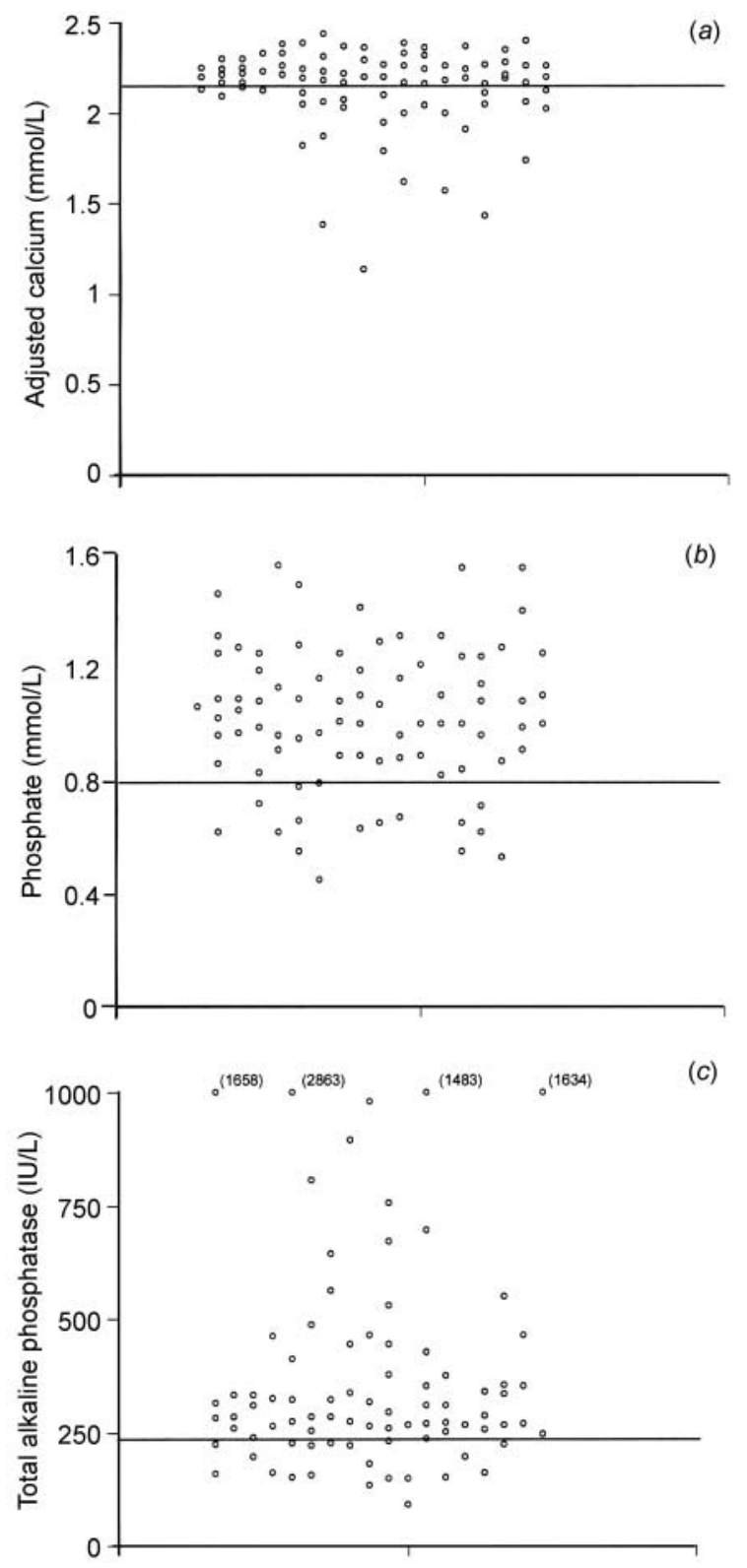

Figure 1 Serum adjusted calcium (a), phosphate (b) and total alkaline phosphatase (c) in patients with 25-OHD deficiency and secondary hyperparathyroidism $(n=84)$ [Horizontal lines indicate lower limits of normal ranges]

OHD $(r=-0.39,-0.56$ to $-0.18 ; P=0.002)$. A borderline positive correlation was found between 25-OHD and calcium $(r=0.22,0$ to $0.42 ; P=0.05)$. 5 patients $(6 \%)$ proved to have coeliac disease- 3 out of the 4 with European origins and 2 of the 80 with Asian origins.

\section{DISCUSSION}

In the context of 25-OHD deficiency, a raised PTH usually indicates secondary hyperparathyroidism (unless there is concomitant primary or tertiary hyperparathyroidism). PTH is the best biochemical marker of underlying histological osteomalacia; ${ }^{7}$ and, at very least, secondary hyperparathyroidism leads to bone resorption and osteoporosis. ${ }^{8}$ This 

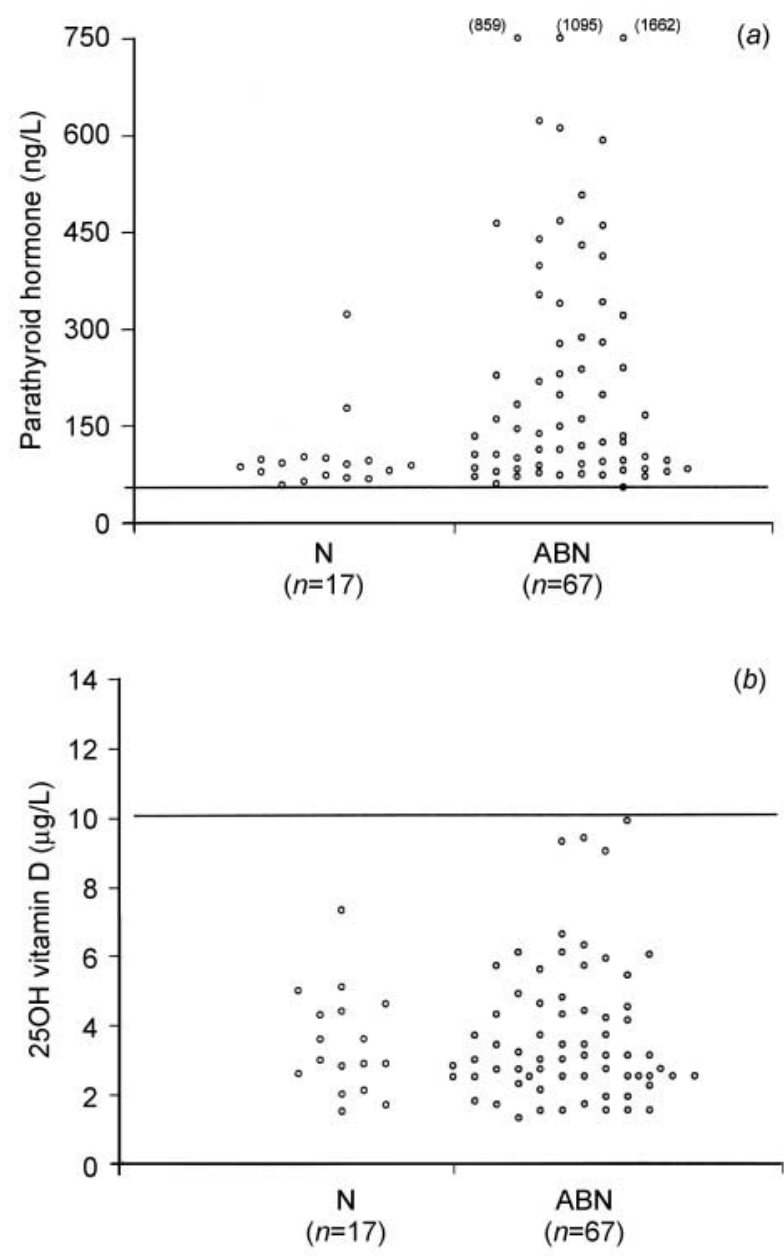

Figure 2 Serum PTH (a) and 25-OHD (b) in patients with normal biochemistry (group $N$ ) and patients with at least one biochemical abnormality (group ABN). Limit of detection for $25-\mathrm{OHD}=1.5 \mu \mathrm{g} / \mathrm{L}$

study of patients with 25-OHD deficiency and secondary hyperparathyroidism shows that $20 \%$ have normal routine biochemistry $(\mathrm{Ca}, \mathrm{PO}$ and ALP) and that, if laboratory testing is confined to routine biochemistry, a substantial minority (20\%) of these high-risk cases will be missed. It may be argued that, since patients were commonly referred to our department because of abnormal biochemistry, this may reflect selection bias. However, in primary care the modes of presentation mean that an even higher proportion with 'biochemical osteomalacia' are likely to escape detection by routine biochemistry. ${ }^{9}$

In Asians, a raised PTH is a better determinant of histological osteomalacia than $25-\mathrm{OHD}^{7,10}$ and the extent of the increase is likely to reflect the severity of bone disease. ${ }^{4}$ In the present study, patients with abnormal routine biochemistry had a greater increase in PTH than those with normal biochemistry - in other words, abnormal routine biochemistry may point to more advanced bone disease. In previous work, only $10 \%$ of patients with histological osteomalacia were found to have normal routine biochemistry, ${ }^{7,11}$ probably because they had more advanced bone disease than our cohort.

Clinical scoring systems have been used to detect osteomalacia and have been suggested as a screening procedure. ${ }^{7,12}$ However, two of the four discriminative criteria used in such a scoring system do not apply to the majority of Asians in Bradford. Whilst health education, professional awareness and appropriate use of supplements should remain the mainstay for prevention of this condition, a biochemical strategy is required where patients present with musculoskeletal symptoms. Although ALP is commonly abnormal in osteomalacia and may predict histological osteomalacia, ${ }^{11}$ total ALP is commonly raised in Asian patients because of obesity or type 2 diabetes mellitus, leading to a high false-positive rate for osteomalacia if used alone. ${ }^{7}$ Routine measurement of PO seems of negligible benefit. Although some workers advocate measurement of 25 -OHD wherever deficiency is suspected, ${ }^{13}$ this is probably a waste of resources in populations with a high prevalence of vitamin D deficiency, since this expensive test may still not indicate whether musculoskeletal symptoms are attributable to vitamin D deficiency. Although the same might be argued for measurement of $\mathrm{PTH}$, it is less costly (about $£ 9$ rather than £25), is more predictive of osteomalacia ${ }^{7,10}$ and is therefore a better initial test for Asian patients with musculoskeletal symptoms. A reasonable policy is to measure $\mathrm{Ca}$ and $\mathrm{PTH}^{7,9,14}$ in the first instance and to presume a diagnosis of vitamin $\mathrm{D}$ deficiency/osteomalacia if PTH is raised in association with a low or low-normal calcium (to avoid inclusion of patients with primary hyperparathyroidism). Measurement of creatinine, endomysial antibodies and mean corpuscular volume (MCV) will detect patients with renal disease and identify patients who should be investigated for possible coeliac disease. Coeliac disease accounted for $2.5 \%$ of Asian cases in this study and should not be forgotten as a cause of osteomalacia in this group. ${ }^{15,16}$

In summary, for populations with a high prevalence of vitamin D deficiency or osteomalacia, biochemical assessment of suspected cases should include measurement of $\mathrm{PTH}, \mathrm{Ca}$, creatinine, FBC and endomysial antibodies. If only routine biochemistry is used, a substantial minority of these high-risk cases will be missed.

Acknowledgments I thank Linda Wood for continuing maintenance of the departmental endocrine database and Doug Hirst for the laboratory assay details.

\section{REFERENCES}

1 Dunnigan MG, Paton JPJ, Hasse S, McNichol GW, Gardner MD, Smith CM. Late rickets and osteomalacia in the Pakistani community in Glasgow. Scott Med J 1962;7:159-67 
2 Ford JA, Colhoun EM, McIntosh WB, Dunnigan MG. Rickets and osteomalacia in the Glasgow Pakistani community, 1961-71. BMJ 1972;ii:677-80

3 Ford JA, McIntosh WV, Butterfield R, et al. Clinical and subclinical vitamin D deficiency in Bradford children. Arch Dis Child 1976;51: 939-43

4 Finch PJ, Ang L, Eastwood JB, Maxwell JD. Clinical and histological spectrum of osteomalacia among Asians in south London. Quart J Med 1992;83:439-48

5 Committee on Medical Aspects of Food Policy. Working Party on the Fortification of Food with Vitamin D. London: HM Stationery Office, 1980

6 Panel on Dietary Reference Values of the Committee on Medical Aspects of Food Policy. Dietary Reference Values for Food Energy and Nutrients for the United Kingdom. London: HM Stationery Office, 1991

7 Nisbet JA, Eastwood JB, Colston KW, et al. Detection of osteomalacia in British Asians: a comparison of clinical score with biochemical measurements. Clin Sci 1990;78:383-9

8 Lips P. Vitamin D deficiency and secondary hyperparathyroidism in the elderly: consequences for bone loss and fractures and therapeutic implications. Endocr Rev 2001;22:477-501
9 Solanki T, Hyatt RH, Kemm JR, Hughes EA, Cowan RA. Are elderly Asians in Britain at a high risk of vitamin D deficiency and osteomalacia? Age Ageing 1995;24:103-7

10 Mawer EB, Backhouse J, Hill LF, et al. Vitamin D metabolism and parathyroid function in man. Clin Sci Molec Med 1975;48:349-65

11 Peach H, Compston JE, Vedi S, Horton LWL. Value of plasma calcium, phosphate and alkaline phosphatase measurements in the diagnosis of histological osteomalacia. J Clin Pathol 1982;35:625-30

12 Peach H, Compston JE, Vedi S. Value of the history in diagnosis of histological osteomalacia among Asians presenting to the NHS. Lancet 1983;ii:1347-9

13 Plehwe WE. Vitamin D deficiency in the 21st century: an unnecessary pandemic? Clin Endocrinol 2003;59:22-4

14 Parfitt AM. Screening for vitamin D depletion. Lancet 1984;i: $227-8$

15 Thalayasingham B. Coeliac disease as a cause of osteomalacia and rickets in the Asian immigrant population. BMJ 1985;290:1146-7

16 Sheikh S, Williamson K, Kearley K, Bassindale S, Lancaster T. Danger of stereotyping in suspected osteomalacia. BMJ 2001;323:149-51 